\title{
Identification of SNPs potentially related to immune responses and growth performance in Litopenaeus vannamei by RNA-seq analyses
}

\author{
Camilla A. Santos ${ }^{\text {Corresp., }}{ }^{1}$, Sónia C.S. Andrade ${ }^{2}$, Patrícia D. Freitas ${ }^{1}$ \\ 1 Departamento de Genética e Evolução, Universidade Federal de São Carlos, São Carlos, São Paulo, Brazil \\ 2 Departamento de Genética e Biologia Evolutiva, Universidade de São Paulo, São Paulo, São Paulo, Brazil \\ Corresponding Author: Camilla A. Santos \\ Email address: camilla.alves@yahoo.com.br
}

Litopenaeus vannamei is one of the most important shrimp species for worldwide aquaculture. Despite this, little genomic information is available for this penaeid and other closely related taxonomic crustaceans. Consequently, genes, proteins and their respective polymorphisms are poorly known for these species. In this work, we used the RNA sequencing technology (RNA-seq) in L. vannamei shrimp evaluated for growth performance, and exposed to the White Spot Syndrome Virus (WSSV), in order to investigate the presence of Single Nucleotide Polymorphisms (SNPs) within genes related to innate immunity and growth performance, both features of great interest for aquaculture activity. We analyzed individuals with higher and lower growth rates; and infected (unhealthy) and non-infected (healthy), after exposure to WSSV. Approximately 7,000 SNPs were detected in the samples evaluated for growth, being 3,186 and 3,978 exclusive for individuals with higher and lower growth rates, respectively. In the animals exposed to WSSV we found about 16,300 unique SNPs, in which 9,338 were specific to non-infected shrimp, and 7,008 were exclusive to individuals infected with WSSV and symptomatic. In total, we describe 4,312 unigenes containing SNPs. About $60 \%$ of these unigenes returned GO blastX hits for Biological Process, Molecular Function and Cellular Component ontologies. We identified 512 KEGG unique KOs distributed among 275 pathways, elucidating the majority of metabolism roles related to high protein metabolism, growth and immunity. These polymorphisms are all located in coding regions, and certainly can be applied in further studies involving phenotype expression of complex traits, such as growth and immunity. Overall, the set of variants raised herein enriches the genomic databases available for shrimp, given that SNPs originated from nextgen are still rare for this relevant crustacean group, despite their huge potential of use in genomic selection approaches. 
1 Identification of SNPs potentially related to immune responses and growth performance in

2 Litopenaeus vannamei by RNA-seq analyses

3

4 Camilla A. Santos ${ }^{1}$, Sónia C.S. Andrade ${ }^{2}$ and Patrícia D. Freitas ${ }^{1}$

5

6 'Departamento de Genética e Evolução, Universidade Federal de São Carlos, São Carlos, SP, 7 Brazil.

$8 \quad{ }^{2}$ Departamento de Genética e Biologia Evolutiva, Instituto de Biociências, Universidade de São 9 Paulo, São Paulo, SP, Brazil.

10

11 Correspondence to: Camilla Alves Santos. Via Washington Luiz km 235, Caixa Postal 676, 12 CEP13565-905, São Carlos-SP, Brazil.

13 Phone: +55-16-3351-8309, Fax: +55 1633518377.

14 E-mail: camilla.alves@yahoo.com.br; patdf@ufscar.br

15

16 Short Title: SNPs from RNA-seq data in L. vannamei 
17 Abstract

18 Litopenaeus vannamei is one of the most important shrimp species for worldwide aquaculture.

Despite this, little genomic information is available for this penaeid and other closely related taxonomic crustaceans. Consequently, genes, proteins and their respective polymorphisms are poorly known for these species. In this work, we used the RNA sequencing technology (RNAseq) in L. vannamei shrimp evaluated for growth performance, and exposed to the White Spot Syndrome Virus (WSSV), in order to investigate the presence of Single Nucleotide Polymorphisms (SNPs) within genes related to innate immunity and growth performance, both features of great interest for aquaculture activity. We analyzed individuals with higher and lower growth rates; and infected (unhealthy) and non-infected (healthy), after exposure to WSSV. Approximately 7,000 SNPs were detected in the samples evaluated for growth, being 3,186 and 3,978 exclusive for individuals with higher and lower growth rates, respectively. In the animals exposed to WSSV we found about 16,300 unique SNPs, in which 9,338 were specific to noninfected shrimp, and 7,008 were exclusive to individuals infected with WSSV and symptomatic. In total, we describe 4,312 unigenes containing SNPs. About $60 \%$ of these unigenes returned GO blastX hits for Biological Process, Molecular Function and Cellular Component ontologies. We identified 512 KEGG unique KOs distributed among 275 pathways, elucidating the majority of metabolism roles related to high protein metabolism, growth and immunity. These polymorphisms are all located in coding regions, and certainly can be applied in further studies involving phenotype expression of complex traits, such as growth and immunity. Overall, the set of variants raised herein enriches the genomic databases available for shrimp, given that SNPs originated from nextgen are still rare for this relevant crustacean group, despite their huge potential of use in genomic selection approaches. 


\section{Introduction}

One of the central challenges in shrimp farming is to avoid economical loss due to animal growth limitations and even population death (Jung et al., 2013; Chen et al., 2015) commonly caused by pathogens. In an attempt of stop pathogen propagation, the organism goes into a severe oxidative metabolic stress yielding many Reactive Oxygen Species (ROS), which may cause cell and DNA structural damages, leading to apoptosis, and consequently to animal death (Rewitz et al., 2006; Qian et al., 2014).

Shrimp and other crustaceans do not own a real adaptive immune system, such as mammals, and are entirely dependent on their innate system, producing a huge variety of immune proteins, such as crustacyanin (Fan et al., 2016) and hemocyanin (Zheng et al., 2016). The antiviral mechanisms and receptors are not well known in crustaceans and some strategies involved in immunity have also been reported, such as nucleic acids that when injected in shrimp organism could be involved in immune gain, as antiviral responses, suggesting a role similar to those assigned to interferons in mammals (Sadler and Williams, 2008; Nehyba et al., 2009; Kongton et al., 2011). Interference-RNAs (RNAi) are also linked to immunity in shrimp, representing a sequence-specific protection against some viral diseases, such as White Spot Syndrome Virus (WSSV) (Xu et al., 2007; Huang and Zhang, 2013; Wang et al., 2013a). Mechanisms acting on defense, such as phagocytosis, are triggered by the pathogen recognition, binding and encapsulation, leading to cell reorganization and subsequent pathogen destruction through mechanisms, such as acid pH (Wongpanya et al., 2007, Wang et al., 2013b; Liu et al., 2014). Apoptosis are also extremely efficient in preventing virus propagation in the organism, eliminating infected cells and providing some possible advantages in host immunity. Moreover, 
63 it is widely known that viral infection and proliferation induces apoptosis in shrimp (Leu et al.,

64 2013, Wang et al., 2013a, Wang et al., 2014). In this way, energy that could be being spent in 65 growth is required by shrimp immunity when exposed to pathogens (Lv et al., 2014; Rao et al., 66 2016). Moreover, external factors, such as temperature and inefficient food supply significantly 67 influences growth performance (Jindra et al., 2013), leading to a not profitable scenario to 68 aquaculture.

Litopenaeus vannamei (Penaeid, Crustacea) is one of the most important shrimp species for worldwide aquaculture, owing a high commercial value aggregated and being an expressive 71 resource for global aquaculture production ( $\mathrm{Lu}$ et al., 2017, Zhao et al., 2017). In the meantime, little information is known about the genes and proteins acting on growth and immune responses 73 of this species (Jung et al., 2013). Before the lack of a reference shrimp genome, the transcriptomic databases have become essential in identifying new molecular variants, and also in providing data regarding proteins involved in growth and immune system issues (Rao et al., 2015; Sun et al., 2015). In this way, RNA-seq approaches, based on the next generation sequencing (nextgen), has enabled the gene sequencing high coverage, allowing the large-scale identification of polymorphisms related to economic characteristics in non-model species, such as crustaceans (Cui et al., 2013, Santos et al., 2014; Yu et al., 2014; Santos et al., 2018). Codominant markers, including Single Nucleotide Polymorphisms (SNPs), are highly informative and abundant in genomes (Brookes, 1999; Vignal et al., 2002) and have been shown to be efficient in linkage and association studies between molecular and phenotypic data in species such as crabs (Cui et al., 2013) and freshwater prawns (Jin et al., 2013). However, studies regarding SNPs identified in penaeids by nextgen (Santos et al., 2014; Baranski et al., 2014; Yu 
85 et al., 2014, Yu et al., 2017; Santos et al., 2018) and other crustaceans are still incipient until 86 present.

Considering this scenario, the present study was conducted aiming to identify SNPs potentially related to immune responses and growth performance in L. vannamei. We analyzed RNA-seq data obtained from individuals with higher and lower growth rates; and infected and non-infected with WSSV, a very aggressive disease in crustaceans that causes enormous losses for shrimp aquaculture industry (Pradeep et al., 2012; Rao et al., 2016; Yu et al., 2017). Our study allowed to identify thousands of specific SNPs in individuals with higher and lower growth rates, and also in healthy and unhealthy shrimps exposed to WSSV. These findings are certainly relevant to be used in further studies considering association of SNPs with immunity and growth performance traits into breeding programs assisted by genomic selection.

\section{Material \& Methods}

\subsection{Biological Sampling}

The biological samples used in the RNA-seq approach are from muscle and hepatopancreas tissues of aquaculture L. vannamei specimens sampled in 2015. We sampled muscle due to its close relation with growth, considering that a faster muscle development may lead to bigger and heavier shrimp, in addition to also be a target for white spot disease (Jung et al., 2013; Shi et al., 2018). On the other hand, hepatopancreas was sampled, given that most of the immune responses in crustaceans occur in hemocytes, which are produced in this tissue, responsible for storing these cells and many proteins involved in the recognition and elimination of pathogens (Guo et al., 2013, Chen et al., 2015). Hemolymph samples for the RNA isolation were also collected from animals exposed to WSSV. However, due to the small size of the 
108 individuals, and the bad health conditions of the animals with WSSV symptoms, we had no 109 success in the subsequent labor steps.

110

\section{1}

Shrimps evaluated for growth performance (growth group)

For this approach, we used Specific Pathogen Free (SPF) L. vannamei pleopod samples genetically improved through a selective breeding program conducted for rapid growth performance and good survival rates in a Brazilian shrimp breeding company. We evaluated 20 families and selected individuals from four families with higher and another four with lower growth rates, respectively, according to the quantitative parameter criteria established for the familiar breeding program implemented by the company (data not available). Muscle tissue samples from a total of 48 shrimps (at 45 days of age), belonging to the eight families, were collected, stored in RNA later (Thermo Fisher Scientific, Waltham, MA, USA), and kept in biofreezer $\left(-80^{\circ} \mathrm{C}\right)$ for the RNA isolation.

\section{Shrimps exposed to WSSV (WSSV group)}

For the WSSV-exposure experiments we sampled shrimps from a Brazilian commercial larviculture laboratory. First, SPF commercial Post-Larvae (PLs) were evaluated to white spot virus by qPCR performed according Silva et al. (2011). The primer pair WSS1011F: 5'TGGTCCCGTCCTCATCTCAG-3' and WSS1079R: 5'-GCTGCCTTGCCGGAAATTA-3' was used in qPCR (OIE, 2017) along with Platinum SYBR®Green qPCR Super Mix UDG kit (Thermo Fisher Scientific, Waltham, MA, USA). After confirming absence or presence of the virus, following recommendations proposed by Pfaffl et al. (2002) and Ririe et al. (1997), negative PLs to WSSV (WSSV-negative) were transported to a farming land tank and exposed to 
131 WSSV. Then, we sampled dozens of unhealthy and healthy shrimps at about two months of age. 132 WSSV symptoms were morphologically detected according Pradeep et al. (2012) and FAO 133 (2018). Hemolymph samples from 20 symptomatic and asymptomatic shrimps were collected to 134 perform WSSV-qPCR tests (Silva et al., 2011; Pfaff et al., 2002; Ririe et al., 1997), and confirm

\subsection{RNA isolation and cDNA Library Construction}

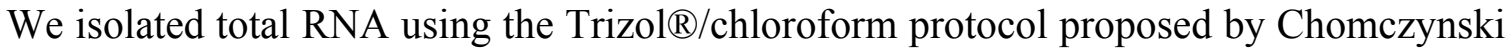
et al. (1995), and checked RNA quality, quantity and integrity in a Q $\mu$ bit fluorometer (Thermo Fisher Scientific), a NanoDrop spectrophotometer (Thermo Fisher Scientific), and a BioAnalyser equipment (Agilent Technologies Inc., Santa Clara, CA, USA), respectively. Samples with RNA Integrity Number $(\mathrm{RIN})>6,0$ were considered proper for later analysis.

We constructed 64 libraries, using a TruSeq RNA Library Preparation V2 kit (Illumina Inc., San Diego, California, USA), for the tissue samples obtained from both growth and WSSV groups. Forty-eight libraries were established for the growth group from (i) 24 muscle samples obtained of animals from the four higher growth families (six shrimps from each family), and (ii) 24 muscle samples of animals from the four lower growth families (six shrimp from each family). Sixteen libraries were established for the WSSV group from (iii) four hepatopancreas samples and (iv) four muscle samples, obtained of four WSSV-positive unhealthy individuals with WSSV symptoms; and (v) four hepatopancreas and (vi) four muscle samples of four 
154 WSSV-negative healthy individuals without WSSV symptoms. All animals exposed to WSSV 155 and selected for the library construction were evaluated by qPCR. The unhealthy animals were 156 all positive to WSSV, and the healthy animals were all negative to the virus. We did not find 157 WSSV-positive healthy animals. A small number of WSSV-positive samples from unhealthy

\subsection{Sequencing, Mapping and SNP identification}

All cDNA libraries were grouped and sequenced on an Illumina HiSeq 2500 Platform (with $2 \times 100$ bp paired-end), using a TruSeq SBS V3 kit (Illumina Inc., Thermo Fisher Scientific). The quality of the raw data generated after sequencing was checked in the FastQC software (version 0.10.1) (http://www.bioinformatics.babraham.ac.uk/projects/fastqc/). All reads were filtered for Phred quality (QS) 23 (sequence average) and 30 (sequence edges), and minimum length of $65 \mathrm{bp}$, using the SeqyClean (v.1.9.9) (https://github.com/ibest/seqyclean). This same software was used to remove contaminant sequences (primers and vectors) listed at the Univec database (https://www.ncbi.nlm.nih.gov/tools/vecscreen/univec/). All reads, available at Sequence Read Archive (SRA-NCBI) under number SRP128934 (BioProject PRJNA428228), were mapped against the reference transcriptome previously de novo assembled for L. vannamei by Santos et al. (2018).

For the mapping, Bowtie2 v.2.2.6 was applied (Langmead and Salzberg, 2012), and the alignment files were used to identify the SNPs. Samtools package (version 1.3) (Li et al., 2009) was used to detect SNPs through the mpileup command, using the following parameters: $-\mathrm{g}-\mathrm{u}$ (calculate the probabilities of the genotypes and generate an uncompressed BCF file), - q20 
177 (minimum mapping quality value for an alignment), -Q20 (minimum quality value of a base), 178 C50 (minimum mapping quality value used to disregard reads with many mismatches), -A (Do not ignore pairs of reads with problems) and -B (do not enable probabilistic realignment, avoiding detection of false SNPs by errors in alignment). SNPs were called through Bcftools (version 1.3) (Li et al., 2009). SNPs with variant base quality $<30$ and sum of the coverage of reads with alternative alleles in the forward and reverse (DP4) strands $<10$ were excluded from the analyzes to avoid artifacts. Only the variants with a minimum of $50 \%$ frequency in each group (higher growth individuals, lower growth individuals, healthy individuals, and unhealthy individuals) were considered for posterior analysis. SNPs with frequency of $50 \%$ or more within the animals of each group were selected for analysis in order to increase the reliability of polymorphisms identified, remaining an adequate number of SNPs for further statistics.

\subsection{SNP Data Statistics and Functional Annotation}

Regarding the unigenes with SNPs only, the -max-missing 0.5 command was used in vcftools (version 0.1.14) (Danecek et al., 2011) to filter polymorphisms with frequency $>=50 \%$. The number of SNPs, transitions and transversions rate (ts:tv) and multi-allelic sites statistics were computed in bcftools using the --stats command for the SNPs of each of the four groups separately. The Minor Allele Frequency (MAF) was calculated in vcftools with maf 0.05 command, with cutoff value of 5\%.

All unigenes, including those with SNPs, were submitted to analysis in the TransDecoder package (http://transdecoder.sourceforge.net/), which was used to identify the contigs candidate coding regions. Meanwhile, Trinotate pipeline (https://trinotate.github.io/) was employed for annotation of the sequences through the following databases: Uniprot (uniref90 + SwissProt) 
200 with cut-off value of $1 \mathrm{e} 10^{-5}$, Gene Ontology (GO) (Ashburner et al., 2000) for the GO terms

201 Biological Process, Molecular Function and Cellular Component and KEGG (Kyoto 202 Encyclopedia of Genes and Genomes) (Kanehisa et al., 2012), with the identification of KOs 203 (KEGG Ortology) followed by the main participating metabolic pathways.

\section{Results}

\subsection{Mapping and SNPs Data}

The 24 libraries constructed from higher growth samples showed 831,930,442 mapped reads, whilst 796,126,113 reads were mapped for the 24 libraries from lower growth samples (96\% of the 1,697,401,992 filtered reads). For the WSSV-exposed group, 191,577,540 and $180,169,952$ reads were mapped for the muscle tissue of healthy and unhealthy shrimps, respectively. For hepatopancreas, we had 200,513,272 and 183,685,654 reads mapped for healthy and unhealthy individuals, respectively. Therefore, 755,946,418 (96\%) reads of samples were mapped against the $786,662,168$ reads after SeqyClean filtering. In this way, 2,484,064,160 reads were mapped against 20,865 unigenes previously to SNP calling. Functional annotation unigenes containing a minimum of five SNPs per unigene (Table S1). Concerning the substitution of bases, the transitions (ts): transversions (tv) rate were of 1.99:1 and 1.97:1 for against the GO base and identification of KEGG pathways in the Trinotate package were also performed (Table 1).

We identified 3,186 and 3,978 exclusive SNPs (frequency $>=50 \%$ ) (Table S1) for the higher and lower growth samples, respectively, including 123 multi-allelic, gathered in 2,300 unigenes (Table 1). The number of SNPs per unigene ranged from 1 to 18 , with $473(20 \%)$ higher and lower growth samples, respectively (Table 2). MAF was calculated for higher and 
223 lower groups highlighting that $98 \%$ of all loci in each group shows the second most common

224 allele with frequency greater than 0.5 in the growth population.

225

226

227

228

229

230

231

232

233

234

235

236

237

238

239

240

241

242

243

244

245

Exclusive SNPs (frequency $>=50 \%$ ) could also be identified for the WSSV-exposed group, highlighting 9,338 and 7,008 variants for healthy and unhealthy shrimps, respectively (Table 1 and Table S1). We found 434 multi-allelic SNPs distributed in 3,807 unigenes. We identified 1 to 42 SNPs/locus for WSSV-exposed shrimps, with 1,027 (27\%) unigenes with five or more SNPs per locus (Table S1). The ts:tv substitution rates were of 1.96:1 and 1.91:1 for healthy and unhealthy samples, respectively (Table 2). Regarding the MAF, healthy and unhealthy animals showed $96 \%$ and $97 \%$ of loci with the second most frequent allele greater than $5 \%$.

\subsection{Uniprot blastX hits on arthropod species}

Following the alignment of sequences against Uniprot database, focus was given on genes showing hits for arthropod species and fitness-related functions, such as growth and disease resistance. Among the higher growth shrimp sampling, the SNPs are mostly located in proteins genes, such as: actin (ACTY), astakine (ASTA) and ryanodine receptor 44 (RY44). On the other hand, for the lower growth sampling our data showed that the SNPs are mostly in genes of chitinase (CHIT3), chitin deacetylase, hemolymph clottable (CLOT) and cuticle (CU17, CUIA and CUPA3) proteins. For the most frequent exclusive SNPs identified in genes from the WSSVexposed shrimps we found SNPs in the heat shock stress 22 (HSP22), 60 (CH60) and 67B2 (HS6B), death-associated inhibitor of apoptosis 1 (IAP1), C-type lectin, crustacyanin (CRA2), crustin, hemocyanin (HCYC) and clottable (CLOT) genes only for healthy animals (Table 3). Exclusive SNPs located in relevant protein genes for all shrimp groups analyzed herein were 
246 identified. The unigenes with more SNPs per loci and with fitness related functions proteins are

247 listed in Table 3 and Table S1.

\subsection{Functional annotation in GO and analysis of metabolic pathways}

After detection of the SNPs, 4,312 unique unigenes containing SNPs (frequency $>=50 \%$ )

251

252

253

254

255

256

257

258

259

260 were established (with hits for any species) and 2,557 returned hits (59\%) against the GO database at level 2, considering both samples evaluated for growth performance and exposure to WSSV. The GO results obtained when the groups were analyzed separately are showed in Figure 1, and also in Supplemental Material for the animals with higher (Table S2) and lower (Table S3) growth performances, and healthy (Table S4) and unhealthy (Table S5) after exposure to WSSV. The main and most frequent ontologies for the shrimps evaluated for growth performance, considering the higher and lower samples, are available in Figure 1 and Table S3. About the WSSV-exposed group, the most relevant ontologies related to fitness are showing in Table S4. Basically, for unhealthy animals the most common Biological Processes identified were the same as those for healthy with chitin binding (GO:0008061) being highlighted as a Molecular Function (Table S5) (Figure 1). When searching for the protein sequences predicted for L. vannamei against the KEGG database, hits were found for 844 (19.5\%) unigenes with SNPs, consisting of 512 unique KEGG Orthologies (KO) distributed in 275 pathways mapped in the KEGG database (Table S6) (Figure 2).

\section{Discussion}

\subsection{BlastX arthropods performance proteins}


Shrimps evaluated for growth performance (growth group)

For animals with higher growth performance we found a greater amount of SNPs in genes, such as actin (ACTY), astakine (ASTA) and ryanodine receptor (RY44) (Table 1 and Table S2). Actin is a classic muscle constituent protein certainly linked to growth. This protein was reported to reach higher mRNA levels in the L. vannamei shrimp during intermolt and premolt periods, suggesting higher growth rates in shrimp abdominal muscle in these stages (Cesar and Yang, 2006). The ryanodine protein is also an important protein acting mainly in the muscle, and its function within the invertebrate group is assumed to be conserved (Maryon et al., 1998). In Drosophila melanogaster, previous reports have shown that when ryanodine receptors are blocked, disruption of muscle contraction occurs (Littleton and Ganetzky, 2000; Sullivan et al., 2000), and consequent impairment of muscle function. Even though these allelic variants could be observed in unigenes among individuals with higher growth rates only, these data can be useful in further studies considering association of these polymorphisms with growth. Additionally, we also found polymorphisms related to cell maintenance and immunity in higher growth performance shrimps. The astakines are cytokines related to hematopoiesis and antiviral immune responses in crustaceans, participating in the signaling of cell-to-cell responses during immune activation (Hsiao and Song, 2009; Cheng et al., 2014).

Therefore, these higher growth performance shrimps seem to be indirectly selected to immune response as well, though this information should be more explored in further studies regarding such approach. The muscle tissue may be a target for many diseases, such as WSSV, and hemocytes are spread all over the crustacean body, what may explain these polymorphisms in immune genes found here. Another point is that animals used in our study are SPF shrimps evaluated for good survival rates too. Thus, finding allele variants that may confer some immune 
292 advantages in individuals with higher growth and survival rates becomes an interesting strategy

293 in increasing productivity (Argue et al., 2002; Cock et al., 2009).

294

295

296

297

298

299

300

301

302

303

304

305

306

307

308

309

310

311

312

313

314

For the lower growth animals, we highlight the polymorphisms observed into chitinase and cuticle genes. Chitinase proteins in general are known for causing the rupture of the bounds between chitin molecules and/or breaking the chitin molecules into smaller sugar carbohydrates. The exoskeleton, which is mostly formed by chitin, is digested during the arthropod molt, allowing the body to grow, at the same time that makes the organism highly vulnerable to external adversities, such as diseases (Proespraiwong et al., 2010). Therefore, a precise balance is required for the animal to grow without being injured. The variants present in CHIT and chitin deacetylase genes, along with the ones in cuticle genes (CU17, CUIA and CUPA3) may be perhaps partially responsible for the lower growth performance of the animals sampled from families with lower growth means. In the case of the exoskeleton takes a longer time to recover after molt, due to chitin formation limitation or chitinase not working efficiently in digesting the cuticle for molt, could be possible reasons for growth deficiency observed in these shrimps (Zhang et al., 2014; Li et al., 2015). However, the allelic variants observed in these genes and their relation in favoring body growth needs to be investigated in details.

\section{Shrimps exposed to WSSV (WSSV group)}

Proteins related to immune activation and responses were the majority identified in the WSSV-exposed and mostly observed in healthy shrimp. The heat shock proteins (HSPs 22, 60 and 67B2) were the most abundant ones. These proteins often act in conditions of severe stress and are triggered when animals are exposed to environmental disturbances (Feder, 1999), showing roles closely linked to proper protein folding and conformation (Kregel et al., 2002; 
315 Tiwari et al., 2015). Thus, polymorphisms located in these protein genes, in healthy animals, 316 may provide some benefit in allowing and keeping the protein conformation, even before stress 317 conditions caused by diseases, resulting in an easier and more efficient manner to maintain cell 318 homeostasis and activate immune responses.

Other relevant genes that presented SNPs in L. vannamei exposed to WSSV and healthy were death-associated inhibitor of apoptosis, hemocyanin, crustin, crustacyanin and lectin protein coding ones. There is a considerable chance that some of the polymorphisms found in this work are related to pathogen recognition inducing phagocytosis, such as lectins (Song et al., 2010) with some variants maybe implying some immunological advantages. In arthropods, one of the most common forms of defense against opportunistic microorganisms is endocytosis with consequent encapsulation and destruction of the pathogen (Wongpanya et al., 2007). Although, endocytosis may be followed by cell apoptosis, what may not be interesting for the animal as whole, given that when an amount of cells are triggered to death all at the same time, the tissue and later the organism, may be injured, leading even to the animal death (Liu et al., 2014). On the other hand, the death-associated inhibitor of apoptosis 1 (IAP1) protein may contribute to reduce the cell damage by acting in apoptosis impairment. As reported in Wang et al. (2013c), when the expression of LvIAP1 gene was knocked down by dsRNA-mediated gene injection, the level of expression of some genes related to WSSV proteins had their levels increased, emphasizing the role of IAP1 in protection against WSSV. In the case of WSSV-infected shrimp it is suggested that IAP1 protein acts preventing apoptosis of infected cells and avoiding the virus to spread even quicker in the organism, as a result of cell lysis (Leu et al., 2012; Wang et al., 2013c). 
against virus, especially WSSV, acting on animal stress and survival responses (Fan et al., 2016), agglutination of the pathogen on hemolymph and cell lysis (Cheng et al., 2008, Shockey et al. 2009; Zheng et al., 2016). The animals used in this work were all obtained from a captive environment and some exposed to a lethal pathogen, what certainly triggers stress responses on their organisms. Thus, the polymorphisms identified in these genes can constitute an important source of information for future studies that evaluate the association of these variants and the immune response.

\subsection{GO and KEGG blastX hits}

When considering the unigenes, returning blastX for all species, with the most allelic variants that compose the GO terms found for animals evaluated for growth performance, a more active metabolism can be observed in higher growth shrimps, with response to oxidative stress, myofibril assembly, muscle development and innate immune responses as the main events occurring at the cell. In animals with lower growth rates, a more basal metabolism seems to predominate, with transcription leading the BP rank. Nonetheless, response to drugs and proteolysis are also observed as frequent events, maybe illustrating the organism acting in an unexpected way before external disturbances except drugs exposure, such as oxidative stress, high population densities and/or few food supply, what demands higher energy waste and stress, leading to limitations in performance.

Regarding the GO terms identified for the WSSV-exposed shrimps, the healthy individuals showed SNPs mainly in genes related to protein folding and actin cytoskeleton organization. These results may show an intense metabolism with a big amount of energy being 
360 required, along with an effective maintenance in protein conformation and function despite the contact with the White Spot Virus, in addition to pathogen endocytosis. In unhealthy animals the events take place more frequently in myofibril assembly and muscle development, together with chitin binding activity. The reason for these GO ontologies may be linked to muscle and exoskeleton cuticle is probably because these are some of the central targets of WSSV in shrimp organism, with the eruption of necrosis white spots in muscle and exoskeleton (Pradeep et al., 2012), reflecting a scenario where the animals are showing to be in a constant attempt of recovering themselves from damages caused by the syndrome.

After KEGG blastX hits, 844 unigenes with 512 and 275 unique KOs and pathways, respectively, were identified. In what regards the number of pathways mapped in the KEGG, the value found here was close to other penaied Illumina transcriptomes, such as in Yu et al., 2014 and Shi et al., 2018, in which 240 for L. vannamei and 295 for Fenneropenaeus chinensis pathways were mapped in KEGG database. These results agreed with the GO annotation previously described here, that highlighted functions related to an intense protein production and metabolism, central roles in redox signaling before external disturbances, such as diseases, and in dealing with not-recognized particles, such as pathogens. The number of KOs in the pathways, such as metabolism, biosynthesis of secondary compounds and ribosome suggests a high carbohydrate metabolism, for the production of energy, and proteins to supply processes as growth and immune defense. The animals evaluated here were submitted to selection for rapid growth, which characterizes the intense demand for proteins and energy for weight gain. However, the distribution of KOs along the mapped pathways varied widely, as can be seen from the number of pathways with correspondence found (Table S6). The absence of a reference genome and the still small number of transcriptome data in crustacean may restrict a more 
383 detailed understanding of the role of some proteins within specific metabolic pathways of more 384 closely related species.

385

386

387 388 389 390 391 392 393

394 395 396 397 398

\section{Conclusions}

The RNA-seq analyses performed herein, using several individuals of L. vannamei evaluated for different performance features of interest for aquaculture enabled the identification of SNPs in important genes related to growth and immunity in crustaceans. In addition, a wide variety of genes with potential fitness-related functions presented several unique SNPs identified for shrimp samples evaluated for (i) higher and (ii) lower growth and (iii) infected (unhealthy) and (iv) non-infected (healthy) after contact with WSSV. Thus, the data generated in this study add relevant information to penaeid transcriptomes, since reports of SNPs detected by nextgen in L. vannamei are still rare in the literature. The RNA sequencing provided a wide coverage allied with high resolution of the generated reads, allowing a significant accuracy, reliability and robustness for the SNPs identified here. These polymorphisms may be potentially applied in high-density chips and high-density linkage maps for Genome Wide Association Studies (GWAS) (Baranski et al., 2014; Yu et al., 2014), providing a base for association analysis between complex traits genotypes and phenotypes. This set of SNPs could be also interesting for allelic-specific expression studies (Bell and Beck, 2009), given that all these polymorphisms are located in coding regions and can directly act in phenotype expression. For that, we recommend specific population validations to confirm the presence of such polymorphisms in other $L$. vannamei populations, including outbreed ones, and also related species.

\section{Acknowledgements}


The authors thank to Centro de Genômica Funcional do Laboratório de Biotecnologia

Animal and Prof. Dr. Luiz Lehmann Coutinho (ESALQ-USP), Piracicaba-SP, Brazil, for the library preparations, sequencing and the computer cluster availability. The authors also thank to Ana Carolina Guerrelhas, Ana Karina Teixeira, Flavio Farias, Karin Kurkjian and João Luis Rocha for supporting the sample collection and providing relevant information related to the fitness performance of the genetically improved shrimp families through a breeding program (private information).

\section{References}

Argue, B. J., Arce, S. M., Lotz, J. M., \& Moss, S. M. (2002). Selective breeding of Pacific white shrimp Litopenaeus vannamei for growth and resistance to Taura Syndrome Virus.

Asai, D. J., \& Koonce, M. P. (2001). The dynein heavy chain: structure, mechanics and evolution. Trends in cell biology, 11(5), 196-202.

Ashburner M., Ball C. A., Blake J.A., Botstein D., Butler H., Cherry J.M., Davis A.P., Dolinski K., Dwight S.S., Eppig J.T., Harris M.A, Hill D.P., Issel-Tarver L., Kasarskis A., Lewis S., M. J. C. (2000). Gene ontology: Tool for the identification of biology. Natural Genetics, 25(may), 25-29.

Baranski, M., Gopikrishna, G., Robinson, N. a, Katneni, V. K., Shekhar, M. S., Shanmugakarthik (2014). The development of a high density linkage map for black tiger shrimp (Penaeus monodon) based on cSNPs. PloS One, 9(1), e85413. https://doi.org/10.1371/journal.pone.0085413

Bell, C. G., \& Beck, S. (2009). Advances in the identification and analysis of allele-specific expression. Genome medicine, 1(5), 56. 
429 Brookes, A. J. (1999). The essence of SNPs. Gene, 234(2), 177-86. Retrieved from $430 \quad$ http://www.ncbi.nlm.nih.gov/pubmed/10395891

Cerenius, L., Lee, B. L., \& Söderhäll, K. (2008). The proPO-system: pros and cons for its role in invertebrate immunity. Trends in Immunology, 29(6), 263-271. http://doi.org/10.1016/j.it.2008.02.009

Cesar, J. R. O., \& Yang, J. (2007). Expression Patterns of Ubiquitin, Heat Shock Protein 70 , aActin and b-Actin Over the Molt Cycle in the Abdominal Muscle of Marine Shrimp Litopenaeus vannamei. Comparative Biochemistry \& Physiology, 559(October 2006), 554559. http://doi.org/10.1002/mrd

Chen, K., Li, E., Li, T., Xu, C., Wang, X., Lin, H.,Chen, L. (2015). Transcriptome and molecular pathway analysis of the hepatopancreas in the Pacific white shrimp Litopenaeus vannamei under chronic low-salinity stress. PLoS ONE, 10(7), 1-22. http://doi.org/10.1371/journal.pone.0131503

Cheng, W., Tsai, I. H., Huang, C. J., Chiang, P. C., Cheng, C. H., \& Yeh, M. S. (2008). Cloning and characterization of hemolymph clottable proteins of kuruma prawn (Marsupenaeus japonicus) and white shrimp (Litopenaeus vannamei). Developmental and Comparative Immunology, 32(3), 265-274. http://doi.org/10.1016/j.dci.2007.05.009

Cock, J., Gitterle, T., Salazar, M., \& Rye, M. (2009). Breeding for disease resistance of Penaeid shrimps. Aquaculture, 286(1-2), 1-11. http://doi.org/10.1016/j.aquaculture.2008.09.011

Cui, J., Wang, H., Liu, S., Qiu, X., Jiang, Z., \& Wang, X. (2014). Transcriptome analysis of the gill of Takifugu rubripes using Illumina sequencing for discovery of SNPs. Comparative Biochemistry and Physiology - Part D: Genomics and Proteomics, 10(1), 44-51. https://doi.org/10.1016/j.cbd.2014.03.001 
452 Danecek, P., Auton, A., Abecasis, G., Albers, C. A., Banks, E., DePristo, M. A., \& McVean, G.

453

454 455

456

457

458

459

460

461

462

463

464

465

466

467

468

469

470

471

472

473

474

(2011). The variant call format and VCFtools. Bioinformatics, 27(15), 2156-2158.

Fan, L., Wang, A., Miao, Y., Liao, S., Ye, C., \& Lin, Q. (2016). Comparative proteomic identification of the hepatopancreas response to cold stress in white shrimp, Litopenaeus vannamei. Aquaculture, 454, 27-34. http://doi.org/10.1016/j.aquaculture.2015.10.016

FAO, Food and Agriculture Organization of the United Nations (2018). http://www.fao.org/fishery/culturedspecies/Penaeus_vannamei/en. Accessed in February $1^{\text {st }}, 2018$.

Feder, M. E., \& Hofmann, G. E. (1999). Heat-shock proteins, molecular chaperones, and the stress response: evolutionary and ecological physiology. Annual review of physiology, 61(1), 243-282.

Gajula M. P., Soni G., Babu G., Rai A., B. N. (2013). Journal of Applied Molecular Interaction Studies of Shrimp Antiviral Protein, PmAV with WSSV RING Finger. Journal of Applied Bioinformatics \& Computational Biology, (2:1), 1-5.

Grabherr, M. G., Haas, B. J., Yassour, M., Levin, J. Z., Thompson, D. A., Amit, I., Chen, Z. (2011). Trinity: reconstructing a full-length transcriptome without a genome from RNASeq data. Nature biotechnology, 29(7), 644.

Guo, H., Ye, C.-X., Wang, A.-L., Xian, J.-A., Liao, S.-A., Miao, Y.-T., \& Zhang, S.-P. (2013). Trascriptome analysis of the Pacific white shrimp Litopenaeus vannamei exposed to nitrite by RNA-seq. Fish \& Shellfish Immunology, 35(6), 2008-16. http://doi.org/10.1016/j.fsi.2013.09.019

Houle, D. (1992). Comparing evolvability and variability of quantitative traits. Genetics, 130(1), 195-204. 
Hsiao, C., \& Song, Y. (2009). A long form of shrimp astakine transcript: Molecular cloning, characterization and functional elucidation in promoting hematopoiesis. Fish and Shellfish Immunology, 28(1), 77-86. https://doi.org/10.1016/j.fsi.2009.10.016

Huang, T., \& Zhang, X. (2013). Host defense against DNA virus infection in shrimp is mediated by the siRNA pathway. European journal of immunology, 43(1), 137-146.

Jin, S., Fu, H., Zhou, Q., Sun, S., Jiang, S., Xiong, Y., Zhang, W. (2013). Transcriptome Analysis of Androgenic Gland for Discovery of Novel Genes from the Oriental River Prawn, Macrobrachium nipponense using Illumina Hiseq 2000. PLoS ONE, 8(10).

Jindra, M., Palli, S. R., \& Riddiford, L. M. (2013). The Juvenile Hormone Signaling Pathway in Insect Development. Annual Review of Entomology, 58(1), 181-204. http://doi.org/10.1146/annurev-ento-120811-153700

Jung, H., Lyons, R. E., Hurwood, D. A., \& Mather, P. B. (2013). Genes and growth performance in crustacean species: A review of relevant genomic studies in crustaceans and other taxa. Reviews in Aquaculture, 5(2), 77-110. http://doi.org/10.1111/raq.12005

Kanehisa, M., Goto, S., Sato, Y., Furumichi, M., \& Tanabe, M. (2012). KEGG for integration and interpretation of large-scale molecular data sets. Nucleic Acids Research, 40(Database issue), D109-14. http://doi.org/10.1093/nar/gkr988

Kregel, K. C., Heat shock proteins: modifying factors in physiological stress responses and acquired thermotolerance (2002). J. Appl. Physiol. (1985), 92(5), 2177-86.

Kongton, K., Phongdara, A., Tonganunt-Srithaworn, M., \& Wanna, W. (2011). Molecular cloning and expression analysis of the interferon- $\gamma$-inducible lysosomal thiol reductase gene from the shrimp Penaeus monodon. Molecular biology reports, 38(5), 3463-3470.

Langmead, B., \& Salzberg, S. L. (2012). Fast gapped-read alignment with Bowtie 2. Nature 
Methods, 9(4), 357-9. http://doi.org/10.1038/nmeth.1923

Leu, J. H., Lin, S. J., Huang, J. Y., Chen, T. C., \& Lo, C. F. (2013). A model for apoptotic interaction between white spot syndrome virus and shrimp. Fish and Shellfish Immunology, 34(4), 1011-1017. https://doi.org/10.1016/j.fsi.2012.05.030

Li, H., Handsaker, B., Wysoker, A., Fennell, T., Ruan, J., Homer, N., \& Durbin, R. (2009). The sequence alignment/map format and SAMtools. Bioinformatics, 25(16), 2078-2079.

Li, X., Cui, Z., Liu, Y., Song, C., \& Shi, G. (2013). Transcriptome analysis and discovery of genes involved in immune pathways from hepatopancreas of microbial challenged mitten crab Eriocheir sinensis. PloS One, 8(7), e68233. https://doi.org/10.1371/journal.pone.0068233

Li, X., Xu, Z., Zhou, G., Lin, H., Zhou, J., Zeng, Q., Gu, X. (2015). Molecular characterization and expression analysis of five chitinases associated with molting in the Chinese mitten crab, Eriocheir sinensis. Comparative Biochemistry and Physiology Part B Biochemistry and Molecular Biology, 187, 110-120. http://doi.org/10.1016/j.cbpb.2015.05.007

Littleton, J. T., \& Ganetzky, B. (2000). Ion channels and synaptic organization: analysis of the Drosophila genome. Neuron, 26(1), 35-43.

Liu, Y., Hou, F., He, S., Qian, Z., Wang, X., Mao, A., Liu, X. (2014). Identification, characterization and functional analysis of a serine protease inhibitor (Lvserpin) from the Pacific white shrimp, Litopenaeus vannamei. Developmental and Comparative Immunology, 43(1), 35-46.

Lu, X., Kong, J., Meng, X., Cao, B., Luo, K., Dai, P., \& Luan, S. (2018). Identification of SNP markers associated with tolerance to ammonia toxicity by selective genotyping from de novo assembled transcriptome in Litopenaeus vannamei. Fish and Shellfish Immunology, 
521

522

73, 158-166.

Lv, J., Liu, P., Gao, B., Wang, Y., Wang, Z., Chen, P., \& Li, J. (2014). Transcriptome analysis of the Portunus trituberculatus: De novo assembly, growth-related gene identification and marker discovery. PLoS ONE, 9(4). http://doi.org/10.1371/journal.pone.0094055

Maryon, E. B., Saari, B., \& Anderson, P. (1998). Muscle-specific functions of ryanodine receptor channels in Caenorhabditis elegans. Journal of Cell Science, 111(19), 2885-2895.

Nehyba, J., Hrdličková, R., \& Bose, H. R. (2009). Dynamic evolution of immune system regulators: the history of the interferon regulatory factor family. Molecular biology and evolution, 26(11), 2539-2550.

OIE - World Organization for Animal Health. Manual of Diagnostic Tests for Aquatic Animals. Chapter 2.2.8. http://www.oie.int/international-standard-setting/aquatic-manual/accessonline/. Accessed in April 2 2018.

Pradeep, B., Rai, P., Mohan, S. A., Shekhar, M. S., \& Karunasagar, I. (2012). Biology, host range, pathogenesis and diagnosis of white spot syndrome virus. Indian Journal of Virology, 23(2), 161-174. https://doi.org/10.1007/s13337-012-0079-y

Proespraiwong P., Tassanakajon A., Rimphanitchayakit V. (2010). Chitinases from the black tiger shrimp Penaeus monodon: phylogenetics, expression and activities. Comparative biochemistry and physiology. Part B, Biochemistry \& molecular biology, 156(2), 86-96.

Qian, X., Ba, Y., Zhuang, Q., \& Zhong, G. (2014). RNA-Seq technology and its application in fish transcriptomics. Omics: A Journal of Integrative Biology, 18(2), 98-110. https://doi.org/10.1089/omi.2013.0110

Rao, R., Bhassu, S., Bing, R. Z. Y., Alinejad, T., Hassan, S. S., \& Wang, J. (2016). A transcriptome study on Macrobrachium rosenbergii hepatopancreas experimentally 
challenged with white spot syndrome virus (WSSV). Journal of Invertebrate Pathology, 136, 10-22. http://doi.org/10.1016/j.jip.2016.01.002

Rao, R., Bing Zhu, Y., Alinejad, T., Tiruvayipati, S., Lin Thong, K., Wang, J., \& Bhassu, S. (2015). RNA-seq analysis of Macrobrachium rosenbergii hepatopancreas in response to Vibrio parahaemolyticus infection. Gut Pathogens, 7(April 2016), 6. http://doi.org/10.1186/s13099-015-0052-6

Rewitz, K. F., Styrishave, B., Løbner-Olesen, A., \& Andersen, O. (2006). Marine invertebrate cytochrome P450: Emerging insights from vertebrate and insect analogies. Comparative Biochemistry and Physiology - C Toxicology and Pharmacology, 143(4), 363-381. https://doi.org/10.1016/j.cbpc.2006.04.001

Ririe, K. M., Rasmussen, R. P., \& Wittwer, C. T. (1997). Product differentiation by analysis of DNA melting curves during the polymerase chain reaction. Analytical biochemistry, 245(2), 154-160.

Santos, C. A., Blanck, D. V., Freitas, P. D. (2014). RNA-seq as a powerful tool for Penaeid shrimp genetic progress. Frontiers in Genetics, 5(August 2014), 1-6.

Santos, C. A., Andrade, S., Teixeira, A. K., Farias, F., Kurkjian, K., Guerrelhas, A. C., Freitas, P. D. (2018). Litopenaeus vannamei Transcriptome Profile of Populations Evaluated for Growth Performance and Exposed to White Spot Syndrome Virus (WSSV). Frontiers in genetics, 9, 120.

Shi, X., Meng, X., Kong, J., Luan, S., Luo, K., Cao, B.,Cao, J. (2018). Transcriptome analysis of "Huanghai No. 2" Fenneropenaeus chinensis response to WSSV using RNA-seq. Fish and Shellfish Immunology, 75(2), 132-138.

Shockey, J. E., O’Leary, N. A., de la Vega, E., Browdy, C. L., Baatz, J. E., \& Gross, P. S. 
(2009). The role of crustins in Litopenaeus vannamei in response to infection with shrimp pathogens: An in vivo approach. Developmental and Comparative Immunology, 33(5), 668673. https://doi.org/10.1016/j.dci.2008.11.010

Song, K. K., Li, D. F., Zhang, M. C., Yang, H. J., Ruan, L. W., \& Xu, X. (2010). Cloning and characterization of three novel WSSV recognizing lectins from shrimp Marsupenaeus japonicus. Fish and Shellfish Immunology, 28(4), 596-603. http://doi.org/10.1016/j.fsi.2009.12.015

Sullivan, K. M., Scott, K., Zuker, C. S., \& Rubin, G. M. (2000). The ryanodine receptor is essential for larval development in Drosophila melanogaster. Proceedings of the National Academy of Sciences, 97(11), 5942-5947.

Sun, S., Xuan, F., Fu, H., Zhu, J., Ge, X., \& Gu, Z. (2015). Transciptomic and histological analysis of hepatopancreas, muscle and gill tissues of oriental river prawn (Macrobrachium nipponense) in response to chronic hypoxia. BMC Genomics, 16, 491. https://doi.org/10.1186/s12864-015-1701-3.

Tiwari, S., Thakur, R., Shankar, J., Tiwari, S., Thakur, R., \& Shankar, J. (2015). Role of HeatShock Proteins in Cellular Function and in the Biology of Fungi. Biotechnology Research International, 2015, 1-11. https://doi.org/10.1155/2015/132635

Tokunaga, F., Yamada, M., Miyata, T., Ding, Y.-L., Hiranaga-Kawabata, M., Muta, T., \& Iwanaga, S. (1993). Limulus Hemocyte Transglutaminase. The Journal of Biological Chemistry, 268(1), 252-261.

Vignal A., Milan D., Sancristobal M., Eggen A. (2002). A review on SNP and other types of molecular markers and their use in animal genetics. Genetics, Selection, Evolution: GSE, 34, 275-305. http://doi.org/10.1051/gse 
590 Wang, Z., Chua, H. K., Gusti, A. a R. a, He, F., Fenner, B., Manopo, I., Kwang, J. (2005).

RING-H2 protein WSSV249 from white spot syndrome virus sequesters a shrimp ubiquitin-conjugating enzyme, PvUbc, for viral pathogenesis. Journal of Virology, 79(14), 8764-8772. https://doi.org/10.1128/JVI.79.14.8764-8772.2005

Wang, X. W., \& Wang, J. X. (2013a). Pattern recognition receptors acting in innate immune system of shrimp against pathogen infections. Fish and Shellfish Immunology, 34(4), 981989. http://doi.org/10.1016/j.fsi.2012.08.008

Wang, P. H., Yang, L. S., Gu, Z. H., Weng, S. P., Yu, X. Q., \& He, J. G. (2013b). Nucleic acidinduced antiviral immunity in shrimp. Antiviral research, 99(3), 270-280.

Wang, P. H., Wan, D. H., Gu, Z. H., Qiu, W., Chen, Y. G., Weng, S. P., He, J. G. (2013c). Analysis of expression, cellular localization, and function of three inhibitors of apoptosis (IAPs) from Litopenaeus vannamei during WSSV infection and in regulation of antimicrobial peptide genes (AMPs). PloS one, 8(8), e72592.

Wang, P. H., Huang, T., Zhang, X., \& He, J. G. (2014). Antiviral defense in shrimp: from innate immunity to viral infection. Antiviral research, 108, 129-141.

Wongpanya, R., Aoki, T., Hirono, I., Yasuike, M., \& Tassanakajon, A. (2007). Analysis of gene expression in haemocytes of shrimp Penaeus monodon challenged with White Spot $\begin{array}{llllll}\text { Syndrome Virus by } & \text { cDNA }\end{array}$ http://doi.org/10.2306/scienceasia1513-1874.2007.33.165

Yu, Y., Wei, J., Zhang, X., Liu, J., Liu, C., Li, F., \& Xiang, J. (2014). SNP discovery in the transcriptome of white Pacific shrimp Litopenaeus vannamei by next generation sequencing. PloS One, 9(1), e87218. http://doi.org/10.1371/journal.pone.0087218

Yu, Y., Liu, J., Li, F., Zhang, X., Zhang, C., \& Xiang, J. (2017). Gene set based association 
analyses for the WSSV resistance of Pacific white shrimp Litopenaeus vannamei. Scientific Reports, 7(January), 40549. https://doi.org/10.1038/srep40549

Xu, J., Han, F., \& Zhang, X. (2007). Silencing shrimp white spot syndrome virus (WSSV) genes by siRNA. Antiviral research, 73(2), 126-131.

Zhang, L., Li, L., Zhu, Y., Zhang, G., \& Guo, X. (2014). Transcriptome analysis reveals a rich gene set related to innate immunity in the Eastern oyster (Crassostrea virginica). Marine Biotechnology (New York, N.Y.), 16(1), 17-33. http://doi.org/10.1007/s10126-013-9526-z

Zhao, W., Wang, L., Liu, M., Jiang, K., Wang, M., Yang, G., Wang, B. (2017). Transcriptome, antioxidant enzyme activity and histopathology analysis of hepatopancreas from the white shrimp Litopenaeus vannamei fed with aflatoxin B1(AFB1). Developmental and Comparative Immunology (Vol. 74). Elsevier Ltd. https://doi.org/10.1016/j.dci.2017.03.031

Zheng, L., Zhao, X., Zhang, P., Chen, C., Liu, S., Huang, R. Zhang, Y. (2016). Hemocyanin from shrimp Litopenaeus vannamei has antiproliferative effect against HeLa cell in vitro. Plos One, 11, e0151801. http://doi.org/10.1371/journal.pone.0151801 
Figure 1

Main Gene Ontology blastX hits for unigenes with SNPs.

Distribution of the GO terms found at level 2 for the categories Biological Process, Molecular Function and Cellular Component $(*)$ for $(A)$ higher and $(B)$ lower growth performance group and (C) healthy and (D) unhealthy WSSV-exposed group unigenes. The y-axis represents the number of loci that compose each GO term and the x-axis the three categories of GO database. Cytoplasm is the most frequent location where cell events take place in all groups (data not shown in chart, but available at Supplementary Material).
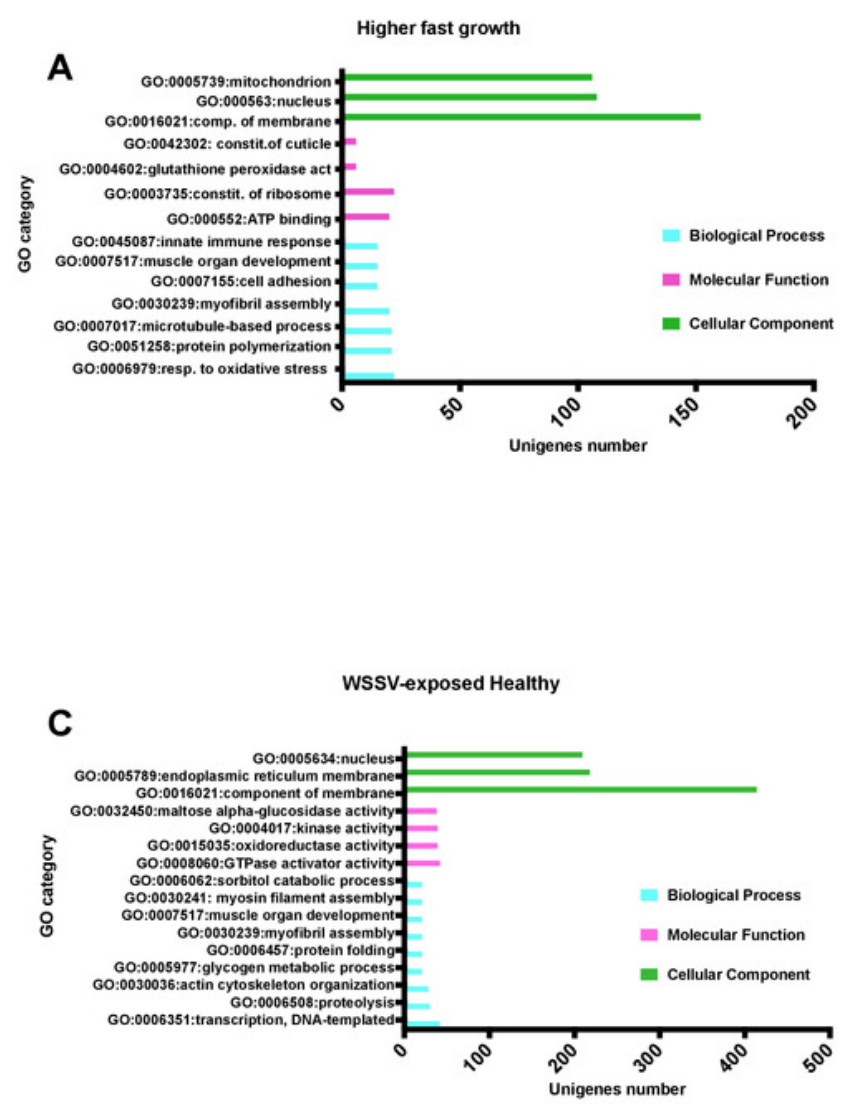
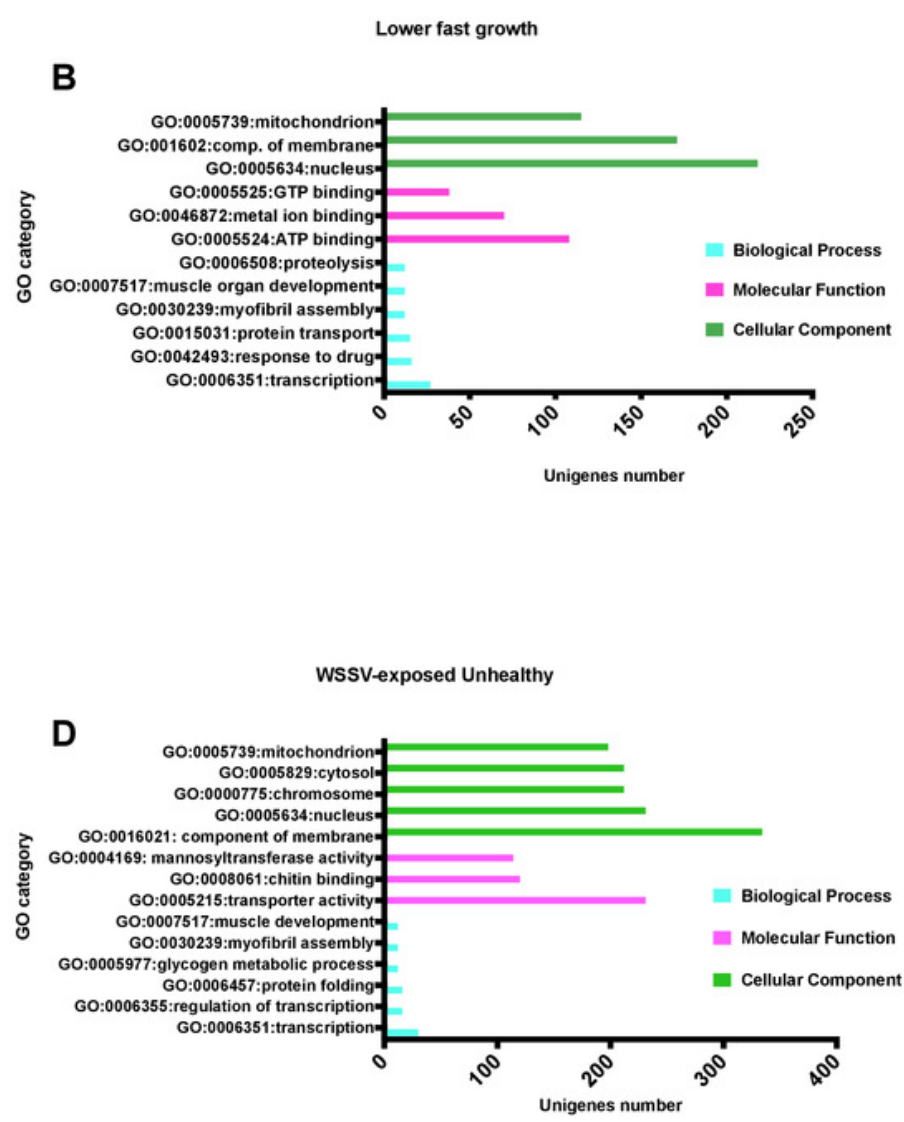


\section{Figure 2}

Some of the KEGG pathways with the most KO number.

Pathways mapped in KEGG for RNA-seq data of Litopenaeus vannamei. The $\mathrm{x}$-axis represents the pathways found and the $y$-axis shows the KO number found in each corresponding pathway. 


\section{Unigenes with SNPs and KEGG blastX hits}

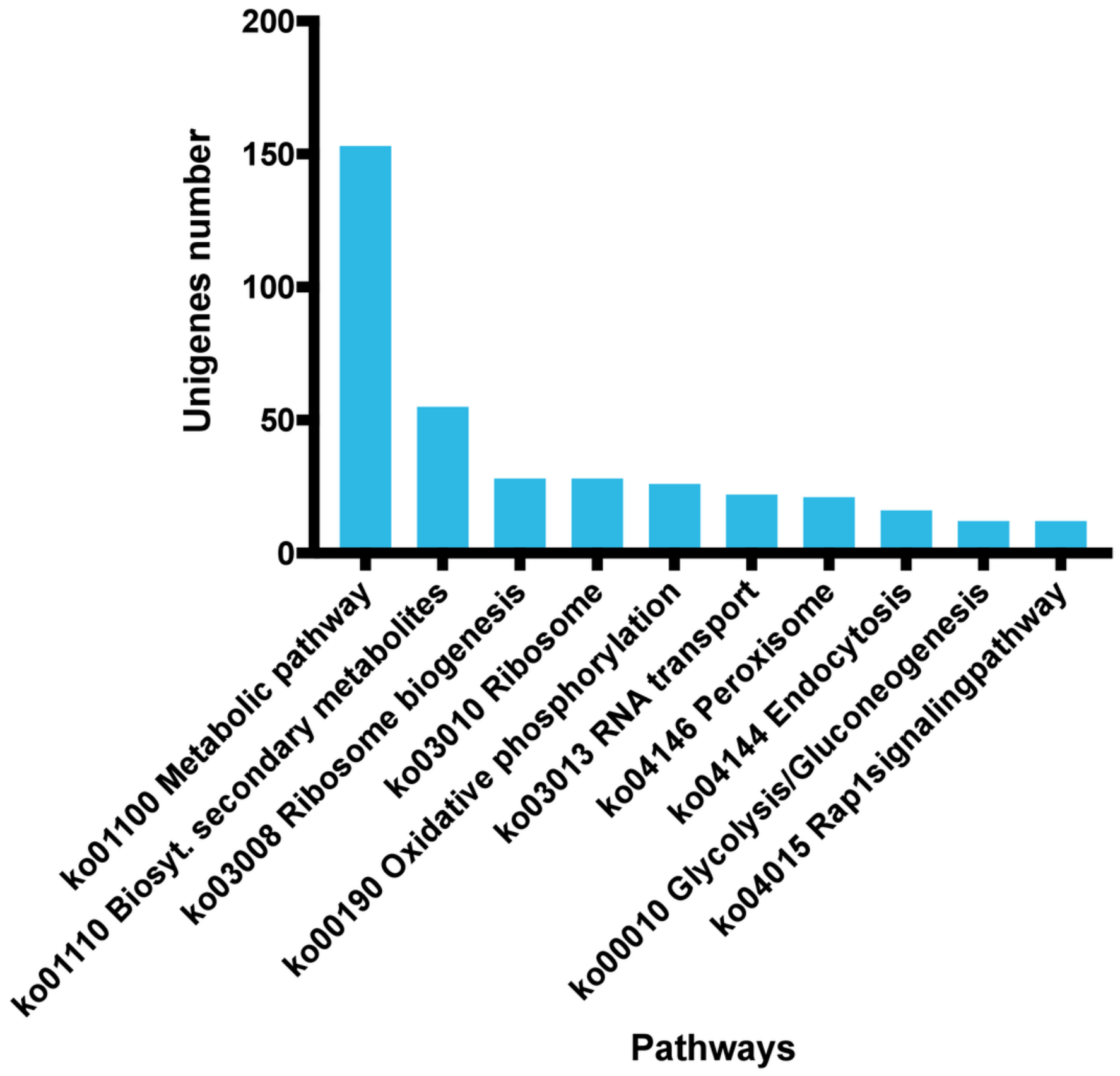




\section{Table $\mathbf{1}$ (on next page)}

Unigenes with SNPs functional annotation.

Overview of SNPs number and functional annotation for the Litopenaeus vannameiRNA-seq data obtained from samples evaluated for growth performance and WSSV-exposure. 


\begin{tabular}{|c|c|c|c|c|}
\hline Not redundant unigenes (trimmed for $>95 \%$ similarity and is & orms). U & $\mathrm{d}$ in SNP & calling. & 20,865 \\
\hline \multicolumn{5}{|c|}{ Trinotate proteins functional annotation } \\
\hline \multicolumn{2}{|c|}{ BlastX hits unigenes (nr) } & \multicolumn{2}{|c|}{$11,256(79 \%)$} & \\
\hline \multicolumn{5}{|c|}{ SNPs (frequency $>=50 \%$ ) } \\
\hline & \multicolumn{2}{|c|}{ Growth } & \multicolumn{2}{|c|}{ WSSV-exposed } \\
\hline & Higher & Lower & Healthy & Unhealthy \\
\hline Unigenes with SNPs & \multicolumn{2}{|c|}{2,300} & \multicolumn{2}{|c|}{3,807} \\
\hline \multirow[t]{2}{*}{$\begin{array}{l}\text { Total unique unigenes with SNPs for growth and WSSV } \\
\text { groups }\end{array}$} & \multicolumn{4}{|c|}{4,312} \\
\hline & Higher & Lower & Healthy & Unhealthy \\
\hline Exclusive SNPs number & 3,186 & 3,978 & 9,338 & 7,008 \\
\hline Total Exclusive SNPs number by group & \multicolumn{2}{|c|}{7,164} & \multicolumn{2}{|c|}{16,346} \\
\hline $\begin{array}{l}\text { Unigenes with GO hits and containing SNPs for growth and } \\
\text { WSSV groups }\end{array}$ & \multicolumn{4}{|c|}{2,557 (59\%) } \\
\hline $\begin{array}{l}\text { Unigenes with KEGG hits and containing SNPs for growth } \\
\text { and WSSV groups }\end{array}$ & \multicolumn{4}{|c|}{$844(20 \%)$} \\
\hline Total unique KOs number & \multicolumn{4}{|c|}{512} \\
\hline Total of unique KEGG metabolic pathways & \multicolumn{4}{|c|}{275} \\
\hline
\end{tabular}




\section{Table 2 (on next page)}

Substitution types statistics found in L. vannamei SNPs.

Transition and transversions rates observed in the SNPs identified on Litopenaeus vannamei transcriptome data obtained for samples evaluated for growth performance and WSSVexposure. 


\begin{tabular}{|c|c|c|c|c|c|c|c|}
\hline \multirow{2}{*}{$\begin{array}{c}\text { Type } \\
\text { Polymorphism }\end{array}$} & \multicolumn{2}{|c|}{ Transitions (ts) } & \multicolumn{5}{|c|}{ Transvertions (tv) } \\
\hline & CT & GA & $\mathbf{A C}$ & AT & GC & GT & $\begin{array}{l}\text { Rate } \\
\text { (ts:tv) }\end{array}$ \\
\hline Higher Growth & $1,127(33,7 \%)$ & $1,096(32,8 \%)$ & $275(8,2 \%)$ & $390(11,6 \%)$ & $200(5,9 \%)$ & $253(7,5 \%)$ & 1.99 \\
\hline Lower Growth & $1,140(33,6 \%)$ & $1,402(32,7 \%)$ & $335(7,8 \%)$ & $516(12 \%)$ & $287(6,7 \%)$ & $303(7 \%)$ & 1.97 \\
\hline WSSV Healthy & $3,479(34,2 \%)$ & $3,262(32 \%)$ & $800(7,8 \%)$ & $1,155(11,3 \%)$ & $662(6,5 \%)$ & $815(8 \%)$ & 1.96 \\
\hline $\begin{array}{c}\text { WSSV } \\
\text { Unhealthy }\end{array}$ & $2,584(32,6 \%)$ & $2,606(32,9 \%)$ & $623(7,8 \%)$ & $988(12,4 \%)$ & $499(6,3 \%)$ & $605(7,6 \%)$ & 1.91 \\
\hline
\end{tabular}




\section{Table 3(on next page)}

Main exclusive SNPs identified in unigenes of higher and lower growth performance group and healthy and unhealthy shrimp after WSSV- exposure.

Loci with the highest number of SNPs exclusive to samples evaluated for growth performance and WSSV-exposed that returned blastX hits for arthropod species. The name of the protein and the number of SNPs/locus are also detailed. 


\begin{tabular}{|c|c|c|c|}
\hline \multirow[t]{3}{*}{ Locus ID } & \multirow[t]{3}{*}{ Protein } & \multirow{2}{*}{\multicolumn{2}{|c|}{$\begin{array}{c}\begin{array}{c}\text { Exclusive SNP } \\
\text { Number }\end{array} \\
\text { Growth } \\
\text { Performance } \\
\end{array}$}} \\
\hline & & & \\
\hline & & Higher & Lower \\
\hline Locus_30356.0|BlastHit|gi|1729925|sp|Q05187.1|TGMH_TACTR & $\begin{array}{c}\text { Hemocyte protein-glutamine } \\
\text { gamma-glutamyltransferase }\end{array}$ & 18 & - \\
\hline Locus_22356.0|BlastHit|gi|728798|sp|P41341.1|ACTY_LIMPO & Actin & 11 & - \\
\hline Locus_30736.0|BlastHit|gi|215273952|sp|Q9W5U2.2|CHIT3_DROME & Chitinase & - & 11 \\
\hline Locus_26398.0 chitin deacetylase 9 precursor [Tribolium castaneum] & Chitin deacetylase & 1 & 10 \\
\hline Locus_28272.0|BlastHit|gi|74897764|sp|Q56R10.1|ASTA_PENMO & Astakine & 10 & - \\
\hline Locus_30850.0|BlastHit|gi|46396031|sp|Q9U572.1|CLOT_PENMO & Clottable & - & 10 \\
\hline Locus_31209.0|BlastHit|gi|33112444|sp|Q24498.3|RY44_DROME & Ryanodine & 10 & - \\
\hline Locus_28234.0|BlastHit|gi|3913391|sp|O02387.1|CU17_BOMMO & Constituent of cuticle & - & 6 \\
\hline Locus_28640.4|BlastHit|gi|3287772|sp|P81384.1|CU1A_HOMAM & Constituent of cuticle & - & 6 \\
\hline Locus_29583.1|BlastHit|gi|5921937|sp|P81577.1|CUPA3_CANPG & Constituent of cuticle & - & 6 \\
\hline $\begin{array}{c}\text { Locus_24086.0 antimicrobial peptide type } 2 \text { precursor IIc } \\
{[\text { Pandalopsis_japonica }]}\end{array}$ & Antimicrobial peptide & - & 5 \\
\hline \multirow[t]{3}{*}{ Locus_29787.0|BlastHit|gi|59797979|sp|Q9W092.1|CHIT2_DROME } & Chitinase & 2 & - \\
\hline & & \multicolumn{2}{|c|}{ WSSV-exposed } \\
\hline & & Healthy & Unhealthy \\
\hline Locus_30670.0|BlastHit|gi|55977856|sp|Q24306.2|IAP1_DROME & $\begin{array}{c}\text { Death-associated inhibitor of } \\
\text { apoptosis } 1 \\
\end{array}$ & 28 & 26 \\
\hline Locus_31211.0|BlastHit|gi|56405335|sp|P37276.2|DYHC_DROME & Dynein & - & 33 \\
\hline Locus_31257.0|BlastHit|gi|152031623|sp|P02515.4|HSP22_DROME & Heat shock 22 & 42 & 5 \\
\hline Locus_31208.0|BlastHit|gi|152031623|sp|P02515.4|HSP22_DROME & Heat shock 22 & 4 & 14 \\
\hline Locus_30588.0|BlastHit|gi|75026464|sp|Q9V895.1|AN32A_DROME & Acidic leucine-rich & 17 & - \\
\hline Locus_31209.0|BlastHit|gi|33112444|sp|Q24498.3|RY44_DROME & Ryanodine & 16 & - \\
\hline $\begin{array}{c}\text { Locus_23466.0 antilipopolysaccharide fator isoform } 5 \\
{[\text { Fenneropenaeus_chinensis }]}\end{array}$ & $\begin{array}{l}\text { Anti-lipopolysaccharide } \\
\text { factor } \\
\end{array}$ & - & 8 \\
\hline Locus_21474.0 C-type lectin [Penaeus_monodon] & Lectin & - & 6 \\
\hline Locus_30118.0|BlastHit|gi|117330|sp|P80007.1|CRA2_HOMGA & Crustacyanin & - & 5 \\
\hline Locus_25051.0|BlastHit|gi|3024418|sp|P81060.1|PEN3C_LITVA & Peneidin & - & 4 \\
\hline Locus_30564.1|BlastHit|gi|122797|sp|P80096.1|HCYC_PANIN & Hemocyanin & 4 & 2 \\
\hline Locus_23573.0 C-type lectin 1 [Marsupenaeus_japonicus] & Lectin & 9 & - \\
\hline Locus_30850.0|BlastHit|gi|46396031|sp|Q9U572.1|CLOT_PENMO & Clottable & 4 & - \\
\hline Locus_25051.0|BlastHit|gi|3024356|sp|P81057.1|PEN2A_LITVA & Peneidin & 1 & - \\
\hline
\end{tabular}

